\title{
Impact of depressed mood on neuropsychological status in temporal lobe epilepsy
}

\author{
S Paradiso, B P Hermann, D Blumer, K Davies, R G Robinson
}

\begin{abstract}
Objectives-Depression is a common psychiatric complication of temporal lobe epilepsy. This study examined the effect of depressed mood on neuropsychological performance among patients with chronic temporal lobe epilepsy.

Methods-Seventy consecutive surgery candidates for medication resistant complex partial seizures of unilateral temporal lobe origin were assessed for psychiatric symptoms and underwent comprehensive neuropsychological assessment.

Results-Standardised psychiatric interview disclosed that $34 \%$ of the patient sample exhibited significant depression. Controlling for seizure frequency, patients with comorbid depression at the time of neuropsychological assessment exhibited significantly poorer performance on measures of intelligence, language, visuoperceptual ability, memory, and executive function. Within lateralised temporal lobe epilepsy groups, the adverse effects of depression on cognitive function were greater in patients with left temporal lobe compared with those with right temporal lobe epilepsy. In addition, depression seemed to be underrecognised and undertreated as none of the patients with epilepsy and comorbid depression were treated for their psychiatric condition at the time of admission for monitoring. Conclusions-Depression, a common psychiatric comorbidity among patients with chronic temporal lobe epilepsy, seems to be undertreated and to have adverse effects on cognitive functioning. (F Neurol Neurosurg Psychiatry 2001;70:180-185)
\end{abstract}

Keywords: depression; neuropsychology; temporal lobe epilepsy

Assessment of neuropsychological status is a standard component of the preoperative assessment of patients with medically intractable complex partial seizures who are candidates for anterior temporal lobectomy. ${ }^{1}$ Neuropsychological assessment is conducted for various purposes including provision of lateralising and localising information as well as for general determination of baseline (preoperative) neurobehavioural status. ${ }^{2}$ Among patients with epilepsy, the degree to which preoperative neuropsychological assessment is influenced by concomitant psychopathology and emotional distress is largely unknown. The estimated prevalence of lifetime to date DSM axis
I disorders among patients with chronic epilepsy is about $60 \%,{ }^{3}$ and prevalence of lifetime to date major depression about $30 \%{ }^{4}{ }^{4}$ Although depressive disorders are widely appreciated to represent a significant comorbid psychiatric complication of chronic epilepsy, ${ }^{36}$ the relation between depressed mood and cognition among patients with epilepsy has not been extensively investigated. ${ }^{7}$ The general psychiatry literature has shown that depression with or without a demonstrable brain lesion can adversely affect a wide range of cognitive abilities. ${ }^{8-15}$

Further, some reports have suggested that the degree of neuropsychological impairment may be mediated by the laterality of lesion. For instance, among depressed patients with a cerebrovascular accident, greater cognitive impairment has been found among depressed than non-depressed patients with left cerebrovascular accident, this relation is not evident among patients with right cerebrovascular accident. ${ }^{14} 15$

It is now widely appreciated in the primary care and psychiatry literature that depression is often underdiagnosed and undertreated. ${ }^{16-18}$ Failure to recognise and treat mood disorders adds to the suffering of patients and their families and reduces health related quality of life. ${ }^{19}$ Although there is general agreement that mood and anxiety disorders are common in epilepsy, the degree to which depression among patients with chronic epilepsy is adequately identified and treated has rarely been examined. The limited evidence to date suggests that there is underrecognition and undertreatment of comorbid psychopathology in general, and depression in particular, among patients with epilepsy. ${ }^{5}$

This investigation therefore examined the effects of interictal comorbid mood disorder on the adequacy of neuropsychological status among patients with chronic unilateral temporal lobe epilepsy. The issue of whether depression exerts greater adverse neuropsychological effects among patients with left versus patients with right temporal lobe epilepsy was examined, as was the degree to which depression had been recognised and treated before presentation for comprehensive evaluation.

Method

PATIENTS

The study sample was a consecutive series of 70 patients who were candidates for anterior temporal lobectomy for treatment of medication resistant complex partial seizures of unilateral temporal lobe origin who met the following criteria: (1) left hemisphere domi- 
Table 1 Demographic, clinical characteristics, and severity of depression

\begin{tabular}{lll}
\hline & Depressed $(n=24)$ & $\begin{array}{l}\text { Non-depressed } \\
(n=46)\end{array}$ \\
\hline Chronological age & $31.6(7.9)$ & $29.0(7.2)$ \\
Sex (\% female) & 66.6 & 33.3 \\
Education (y) & $12.4(2.3)$ & $13.1(2.4)$ \\
Handedness (\% right handed) & 91.9 & 89.1 \\
Age at onset (y) & $11.3(10.9)$ & $10.0(9.9)$ \\
Laterality of TLE (\% left origin) & 62.5 & 69.5 \\
Hippocampal sclerosis (\% moderate to marked) & 76.7 & 63.1 \\
Seizure frequency (number/month) & $65.8(125.8)^{\star}$ & $17.5(43.1)$ \\
Patients on two or more AEDs & 8.3 & 18.1 \\
CES-D & $18.2(10.5) \dagger$ & $10.7(8.23)$ \\
BDI & $11.0(7.24) \ddagger$ & $6.0(6.67)$ \\
MMPI-D & $72.4(13.9) \S$ & $61.3(14.5)$ \\
\end{tabular}

Values are means (SD) or percentage of subjects. BDI=Beck depression inventory; CES-D =Centre for Epidemiological Studies-depression scale, MMPI-D=Minnesota multiphasic personality inventory depression subscale. ${ }^{\star} t(62)=2.26, \mathrm{p}<0.03 ; \dagger F(1,63)=8.8, \mathrm{p}<.005$; $\ddagger F(1,61)=5.5, \mathrm{p}<0.025 ; § F(1,62)=9.3, \mathrm{p}<0.004$.

nant for speech as determined by bilateral intracarotid sodium amobarbital testing (Wada test $\left.^{20}\right) ;(2)$ patients with tumours, cysts, cortical dysgenesis, encephalomalacia, and other lesions disclosed by MRI were excluded. The primary MRI finding was hippocampal atrophy, but there were a few with comorbid cerebellar atrophy; (3) WAIS-R full scale IQ $>69$; and (4) chronological age $>17$ years. All patients underwent continuous ( 24 hour) EEG monitoring of spontaneous seizures with scalp and then subdural strip electrodes. ${ }^{21}$ Generally, three spontaneous seizures were recorded with invasive EEG procedures, and localisation of ictal onset was determined by the electroencephalographer, blinded to the results of the neuropsychological assessment. The monitoring period lasted about 1 week. The psychiatrist and neuropsychologist conducted their evaluations independently, and both were blinded to the EEG findings and the results of each others' assessments until the final consensus conference.

NEUROPSYCHIATRIC EXAMINATION

All patients underwent a comprehensive standardised psychiatric examination supplemented by procedures developed specifically to assess personality and behavioural characteristics of patients with epilepsy. ${ }^{4}$ All patients underwent a semistructured psychiatric interview (with DB) and a next of kin was interviewed to verify the completeness and accuracy of the information. Patients and next of kin jointly completed the epilepsy questionnaire and, separately, a modified form of the neurobehavioural inventory. ${ }^{22}$ The epilepsy questionnaire focused on mood and mood lability, energy, sleep disturbances, atypical pain, irritability, anxiety, specific fears, paranoid ideation, hallucinatory experiences, confusional episodes in the absence of seizures, and various personality characteristics (for example, viscosity, good heartedness, religiosity). In addition, to assess the severity of self rated depressed mood patients were administered the Beck depression inventory (BDI), ${ }^{23}$ the Centre for Epidemiological Studies depression scale, ${ }^{24}$ and the Minnesota multiphasic personality inventory (MMPI) ${ }^{25}$ These data were not used by the psychiatrist in his determination of mood state.
NEUROPSYCHOLOGICAL EXAMINATION

All patients underwent a comprehensive neuropsychological evaluation that included measures of psychometric intelligence (WAIS-R verbal, performance, and full scale $\mathrm{IQ}^{26}$ ), language function (WRAT-R reading, ${ }^{27} \mathrm{MAE}$ visual naming ${ }^{28}$ - number correct, and token tes $^{29}$ - number correct); visuoperceptual and visuospatial abilities (judgement of line orientation $^{30}$ - number correct, facial recognition test $\mathrm{t}^{30}$-number correct); verbal memory (WMS logical memory ${ }^{31}$ - number of units on immediate recall and \% retained after $30 \mathrm{~min}-$ utes, Warrington word recognition ${ }^{32}$ - number correct); visual memory (WMS visual reproduction $^{31}$-number of units on immediate recall and \% retained after 30 minutes, Warrington face recognition ${ }^{32}$ - number correct); executive functions (trail making test $(B)^{33}$ - time completed, Wisconsin card sort ${ }^{34}$ - perseverative responses, controlled oral word association $^{35}$ - number of words); and psychomotor/attention (trail making test $-\mathrm{A}^{33}$ - time completed). No subjects were on antidepressant medication at the time of neuropsychological testing.

\section{NEUROPATHOLOGY}

Surgical specimens were obtained according to methods described elsewhere. ${ }^{36}$ Pathology was graded according to a standardised system providing ratings of the degree of hippocampal sclerosis from none or mild (grade 0) to marked (grade 4 ). ${ }^{21}{ }^{36}$

\section{STATISTICAL ANALYSIS}

Continuous variables were analyzed using $t$ tests, analysis of variance (ANOVA), and analysis fo covariance (ANCOVA). Discrete variables were analyzed using likelihood ratio and Wald $\chi^{2}$ tests. Given the number of experiment-wise comparisons the $\alpha$ level was set at 0.05 despite the directional hypothesis of the investigation (depressed patients expected to show poorer cognitive performance).

\section{Results}

DEMOGRAPHIC AND CLINICAL CHARACTERISTICS Semistructured psychiatric interview showed that $24(34 \%)$ patients exhibited depressed mood and $46(66 \%)$ did not. Demographic and clinical characteristics of depressed and nondepressed patients are shown in table 1. Female patients exhibited a twofold increase in depressed mood compared with male patients (female $66.7 \% \quad v$ male $33.3 \%$ ) but this difference failed to reach statistical significance (likelihood ratio $\chi^{2}(1)=2.83, \mathrm{p}<0.10$ ).

There were no significant differences between the depressed and non-depressed patients in chronological age, education, handedness, patient's age at seizure onset, and laterality of temporal lobe seizure onset (table 1). There was no significant relation between depression and antiepileptic drug monotherapy versus polytherapy (likelihood ratio $\left.\chi^{2}(1)=3.56, p>0.1\right)$.

Seizure frequency was higher in depressed than in non-depressed patients (table 1). Seizure frequency in the left temporal lobe epi- 
Table 2 Symptoms accompanying depressive mood

\begin{tabular}{lll}
\hline & Depressed & Non-depressed \\
\hline Anergia & $17(70.8)^{\star \star \star}$ & $9(19.5)$ \\
Sleep disturbances & $10(41.6)^{\star \star}$ & $4(8.7)$ \\
Pain & $12(50.0)^{\star}$ & $8(17.3)$ \\
Euphoria & $7(29.1)$ & $7(15.2)$ \\
Irritability & $16(66.6)^{\star \star}$ & $12(26.0)$ \\
Anxiety & $14(58.3)^{\star \star \star}$ & $3(6.52)$ \\
Specific phobias & $8(25)^{\star \star}$ & 0 \\
\hline Values are No (\%) of patients with & positive symptoms, \\
${ }^{\star} \mathrm{p}<0.005 ;{ }^{\star \star} \mathrm{p} \leqslant 0.001 ;{ }^{\star \star \star} \mathrm{p}<0.00001$. &
\end{tabular}

lepsy group did not differ significantly between the depressed and non-depressed patients $(p>0.1)$ whereas in the right temporal lobe epilepsy group the depressed patients exhibited significantly poorer seizure frequency compared with non-depressed patients $(\mathrm{p}<0.05)$.

\section{SEVERITY OF DEPRESSION}

Patients with clinically assessed and determined depressed mood exhibited significantly worse self reported depressive symptoms on the CES-D, BDI, and MMPI depression scale (table 1). Two factor ANOVAs (depressed/ non-depressed mood and left/right temporal lobe seizure onset) were computed for the CES-D, BDI, and MMPI-D. Results yielded significant main effects of depression across all measures (CES-D $F(1,63)=8.8, \mathrm{p}<0.005$; BDI $F(1,61)=5.5, \mathrm{p}<0.025 ;$ MMPI-D $F(1$, $62)=9.3, p<0.004)$ and no significant side or interaction effects (all $\mathrm{p}>0.2)$. There were no significant laterality effects for the CES-D (left temporal $=12.8$ (9.4), right temporal $=13.9$ (10.1), $t(61)=0.43, \mathrm{p}>0.5)$, BDI $(\mathrm{left}=6.73$ $(6.2)$, right $=9.17(8.5), t(59)=1.28, \mathrm{p}>0.2)$, or MMPI-D scale $(\mathrm{left}=64.2$ (14.3), right $=65.8$ (16.9), $t(60)=0.4, \mathrm{p}>0.7)$. Patients with clinically determined depressed mood exhibited significantly rates of lack of energy, poor sleep, pain, irritability, anxiety, and phobias (table 2). All patients had been followed up by their referring physicians for treatment of chronic epilepsy, but depression had not been recog-

Table 3 Neuropsychological status in depressed and non-depressed groups

\begin{tabular}{|c|c|c|}
\hline Domains & Depressed $(n=24)$ & Non-depresssed $(n=46)$ \\
\hline \multicolumn{3}{|l|}{ Psychometric intelligence: } \\
\hline Full Scale IQ & $82.2(9.2)^{\star}$ & $89.4(11.8)$ \\
\hline Verbal IQ & $84.0(9.8) \dagger$ & $90.1(12.5)$ \\
\hline Performance IQ & $82.6(10.4) \ddagger$ & $90.5(12.8)$ \\
\hline \multicolumn{3}{|l|}{ Language: } \\
\hline WRAT-R Word reading & $82.7(15.7)$ & $86.9(15.1)$ \\
\hline Token test & $39.8(3.8) \S$ & $41.9(2.4)$ \\
\hline Visual naming & $43.0(8.8)$ & $48.4(7.6)$ \\
\hline \multicolumn{3}{|l|}{ Visuospatial } \\
\hline Judgement of line orientation & $21.7(5.3)^{\star \star}$ & $24.0(4.7)$ \\
\hline Facial recognition & $44.1(5.6)$ & $45.6(3.8)$ \\
\hline \multicolumn{3}{|l|}{ Memory/learning: } \\
\hline WMS Logical memory (immediate) & $10.0(6.5)$ & $11.5(6.3)$ \\
\hline M & $47.7(31.5)+\dagger$ & $62.7(27.4)$ \\
\hline RMT Word recognition & $39.5(6.1)$ & $40.4(5.8)$ \\
\hline WMS Visual reproduction (immediate) & $5.2(2.4) \ddagger \ddagger$ & $7.2(2.5)$ \\
\hline WMS Visual reproduction ( $\%$ retained) & $66.8(32.8)$ & $75.9(24.7)$ \\
\hline RMT Face recognition & $36.8(6.3)$ & $38.7(4.8)$ \\
\hline \multicolumn{3}{|l|}{ Psychomotor/attention: } \\
\hline Trail making test $(\mathrm{A})$ & $40.2(15.7)$ & $35.5(11.8)$ \\
\hline \multicolumn{3}{|l|}{ Executive function: } \\
\hline Controlled oral word association & $27.8(5.9) \sqrt{\S}$ & $32.7(9.7)$ \\
\hline Trail making test (B) & $91.8(43.2)$ & $75.7(28.3)$ \\
\hline $\begin{array}{l}\text { Wisconsin card sort test (perseverative } \\
\text { responses) }\end{array}$ & $22.1(17.2)$ & $18.5(21.7)$ \\
\hline
\end{tabular}

Values are means (SD) scores. ${ }^{\star} t(68)=2.57, \mathrm{p}<0.01 ; \dagger t(68)=2.08, \mathrm{p}<0.05 ; \ddagger t(68)=2.59, \mathrm{p}<0.01 ;$ $\$ t(68)=2.71, \mathrm{p}<0.009 ; \Phi t(68)=2.65, \mathrm{p}<0.01 ;{ }^{\star \star} t(68)=1.83, \mathrm{p}<0.08 ;+\dagger t(68)=2.05, \mathrm{p}<0.05 ;$ $\ddagger \ddagger t(68)=3.15, \mathrm{p}<0.003 ; \int \oint t(68)=2.23, \mathrm{p}<0.04 ; \uparrow \uparrow t(68)=1.87, \mathrm{p}<0.07$. nised or treated as reflected by lack of antidepressant medication therapy. At the end of the monitoring period, 20 of the 24 patients with depressed mood $(83.3 \%)$ required and were treated with antidepressant medication.

\section{NEUROPATHOLOGY}

A surgical specimen adequate for grading of hippocampal pathology was obtained from 63 patients. There was no significant relation between degree of mesial temporal lobe sclerosis and depressed mood (Wald $\chi^{2}(4)=0.5$, $\mathrm{p}>0.7$ ), side of focus (Wald $\chi^{2}(4)=2.2, \mathrm{p}>0.9$ ), or their interaction (Wald $\chi^{2}(4)=2.6, \mathrm{p}>0.6$ ).

NEUROPSYCHOLOGICAL ASSESSMENT

Comparisons between depressed/nondepressed patients across the neuropsychological battery are shown in table 3. ANCOVA (with seizure frequency as a covariate) showed that depressed patients exhibited significantly lower performance on the summary IQ measures (WAIS-R FSIQ $(\mathrm{p}<0.006)$, VIQ $(p<0.03)$, PIQ $(p<0.004))$, language function (visual naming $(\mathrm{p}<0.006)$, token test $(p<0.009)$ ), visuoperceptual ability (judgement of line orientation $(\mathrm{p}<0.03)$ ), memory function (visual reproduction, \% retained $(p<0.001))$, and psychomotor speed/ alternation (trails A $(p=0.05)$, trails B $(p<0.03))$. Similar trends were evident for additional measures of memory, language, and visuoperceptual ability (logical memory, \% retained $(p<0.10))$, verbal fluency $(p<0.06)$, facial recognition test $(\mathrm{p}<0.06))$, but these differences did not reach statistical significance.

Additional analyses were performed to compensate for possible data deviation from linearity. Comparisons between depressed/nondepressed patients were performed after subjects were equated for seizure frequency. Non-depressed patients with less than five seizures/month and three patients with seizure frequency greater than 270/month (outlier Mahalanobis distance $>5$ ) were excluded. These selection criteria yielded 22 depressed (mean seizure frequency 31.9 (SD 55.0)) and 24 non-depressed patients (19.0 (SD 19.8)) $(t(42)=-1.0, \mathrm{p}>0.3)$. Results (available in detail from the authors) showed that depressed patients had significantly poorer performance across measures of verbal and non-verbal psychometric intelligence, language, visual memory, and executive abilities, consistent with the prior results.

Neuropsychological performance was then examined within the left and right temporal lobe groups (table 4). When seizure frequency was subjected to covariance analysis, in the left temporal lobe epilepsy group, depressed patients, compared with non-depressed patients, exhibited significantly lower WAIS-R FSIQ, PIQ $(\mathrm{p}<0.004)$, and VIQ $(\mathrm{p}<0.015)$ scores, language scores (token test $(\mathrm{p}<0.001)$, visual naming $(\mathrm{p}<0.006))$, visuoperceptual and spatial performance (judgement of line orientation, facial recognition (both $\mathrm{p}$ values $<0.03$ )), complex psychomotor processing (trails B $(\mathrm{p}<0.03)$ ), visual memory (visual reproduction-immediate $(\mathrm{p}<0.015))$, and 
Table 4 Neuropsychological status in depressed and non-depressed left and right temporal lobe groups

\begin{tabular}{|c|c|c|c|c|}
\hline \multirow[b]{2}{*}{ Domains } & \multicolumn{2}{|l|}{ Left temporal } & \multicolumn{2}{|l|}{ Right temporal } \\
\hline & $\begin{array}{l}\text { Depressed } \\
(n=15)\end{array}$ & $\begin{array}{l}\text { Non-depressed } \\
(n=32)\end{array}$ & $\begin{array}{l}\text { Depressed } \\
(n=9)\end{array}$ & $\begin{array}{l}\text { Non-depressed } \\
(n=14)\end{array}$ \\
\hline \multicolumn{5}{|l|}{ Psychometric intelligence: } \\
\hline Full scale IQ & $79.2(7.1)^{\star}$ & $89.0(10.7)$ & $87.2(10.7)$ & $90.2(14.5)$ \\
\hline Verbal IQ & $80.8(8.5) \dagger$ & $89.2(10.6)$ & $89.3(9.8)$ & $92.1(16.5)$ \\
\hline Performance IQ & $80.2(8.6) \ddagger$ & $91.0(12.1)$ & $86.6(12.5)$ & $89.5(14.7)$ \\
\hline \multicolumn{5}{|l|}{ Language: } \\
\hline WRAT-R Word reading & $80.7(15.7)$ & $86.4(15.0)$ & $86.1(16.0)$ & $88.2(16.1)$ \\
\hline Naming & $41.2(8.5) \S$ & $47.3(7.6)$ & $46.2(8.8)$ & $51.1(7.2)$ \\
\hline Token test & $38.8(4.1)$ & $41.9(2.3)$ & $41.5(2.7)$ & $41.7(2.6)$ \\
\hline \multicolumn{5}{|l|}{ Visuospatial: } \\
\hline Judgment of line orientation & $21.6(5.3)^{\star \star}$ & $24.0(3.7)$ & $21.8(5.7)$ & $24.0(6.5)$ \\
\hline Facial recognition & $43.0(5.9)+\dagger$ & $45.7(4.2)$ & $46.0(4.6)$ & $45.4(2.9)$ \\
\hline \multicolumn{5}{|l|}{ Memory/learning: } \\
\hline WMS Logical memory (immediate) & $8.0(5.8)$ & $10.2(5.7)$ & $13.3(6.6)$ & $14.3(6.9)$ \\
\hline WMS Logical memory (\% retained) & $48.4(32.9)$ & $56.0(28.8)$ & $46.6(31.1)+\dagger+$ & $78.0(15.9)$ \\
\hline RMT Word recognition & $38.9(5.6)$ & $40.5(4.9)$ & $40.5(7.1)$ & $40.3(7.8)$ \\
\hline WMS Visual reproduction (immediate) & $5.4(2.4) \neq \ddagger$ & $7.3(2.3)$ & $4.7(2.5)$ & $6.8(3.0)$ \\
\hline WMS Visual reproduction (\% retained) & $73.0(33.5)$ & $78.4(22.8)$ & $56.6(30.8)$ & $70.1(28.7)$ \\
\hline RMT Face recognition & $37.0(4.9)$ & $39.1(4.7)$ & $36.4(8.5)$ & $37.7(5.2)$ \\
\hline \multicolumn{5}{|l|}{ Psychomotor/attention: } \\
\hline Trail making test $(\mathrm{A})$ & $37.8(15.0)$ & $34.2(12.7)$ & $44.2(16.2)$ & $38.3(9.36)$ \\
\hline \multicolumn{5}{|l|}{ Executive abilities: } \\
\hline Controlled oral word association & $27.6(4.23) 85$ & $32.2(9.9)$ & $28.2(8.2)$ & $33.8(9.5)$ \\
\hline Trail making test $(\mathrm{B})$ & $95.8(52.2) 99$ & $73.3(25.7)$ & $85.1(22.5)$ & $81.0(33.8)$ \\
\hline Wisconsin card sort test (perseverative responses) & $26.5(17.1)^{\star \star \star}$ & $15.9(13.3)$ & $14.8(15.6)$ & $24.5(34.1)$ \\
\hline
\end{tabular}

Values are means (SD). Intralobe $t$ tests: Left: ${ }^{\star} t(45)=3.20, \mathrm{p}<0.003 ; \dagger t(45)=2.69, \mathrm{p}<0.0 ; \ddagger t(45)=3.08, \mathrm{p} 0<.004 ; \$ t(45)=3.28$, $\mathrm{p}<0.003 ; \Phi t(45)=2.45, \mathrm{p}<.01 ;{ }^{\star \star} t(45)=1.76, \mathrm{p}<0.09 ; \dagger+t(45)=1.81, \mathrm{p}<0.08 ; \ddagger \neq t(45)=2.55, \mathrm{p}<0.01 ; \mathbb{S S t}(45)=1.70, \mathrm{p}<0.1 ; \Phi \uparrow t$ $(45)=1.98, \mathrm{p}<0.05 ;{ }^{\star \star \star} t(45)=2.31, \mathrm{p}<0.03$. Right: $\dagger+\dagger t(21)=3.19, \mathrm{p}<0.004$.

problem solving (WCST perseverative responses $(p<0.007))$. Compared with nondepressed patients with right temporal lobe epilepsy, depressed patients in the same group showed fewer differences with lower memory scores (WMS logical memory, \% retained $(\mathrm{p}<0.015)$, and a trend for poorer visual reproduction, $\%$ retained $(p<0.06))$. As neurocognitive data were analyzed across groups of differing sizes, we also examined side by depressed mood interactions controlling for seizure frequency. The measures that reached statistical significance for the interaction were the token test $(F(1,61)=6.77, \mathrm{p}<0.012)$ and Wisconsin card sort perseverative responses $(F(1$, $61)=4.32, \mathrm{p}<0.05)$. Facial recognition showed a non-significant trend $(F(1,61)=3.85$, $\mathrm{p}<0.097)$. Using samples equated for seizure frequency yielded essentially the same results.

\section{Discussion}

The present study examined the effects of depressed mood on cognition among patients with lateralised temporal lobe epilepsy. The following are the salient findings.

Firstly, depressed mood was associated with significant adverse effects across a wide range of neuropsychological domains including general intelligence, language, visuoperceptual abilities, memory, and executive functioning. There was a significant difference in seizure frequency between the depressed versus nondepressed patients in the right but not left temporal lobe epilepsy group, and when seizure frequency was accounted for with the use of two differing statistical approaches, the adverse effects of depressed mood on cognition remained evident. Thus, consistent with the more general neuropsychological and neuropsychiatry literature, depression seems to be associated with decreased efficiency of cognitive functioning among patients with unilateral temporal lobe epilepsy. To our knowledge, this is the first report to demonstrate that comorbid depression is associated with poorer neuropsychological status in temporal lobe epilepsy. Whether this relation between mood state and cognition influences the lateralising ability of the neuropsychological examination remains to be determined. The degree to which comorbid psychiatric disorder affects the specificity and sensitivity of neuropsychological procedures to lateralised temporal lobe lesions is an issue of clinical relevance.

Secondly, whereas there was no difference in the frequency of depression in left versus right temporal lobe epilepsy groups, the effects of depression on neuropsychological status seemed to be somewhat accentuated in patients with left compared with those with right temporal lobe epilepsy. These findings need to be interpreted with caution due to differing group sizes in our sample. Studies with larger sample sizes are needed to determine whether depressed compared with non-depressed left temporal lobe patients exhibit greater impairment in cognition (for example, general intelligence, executive, language, visuoperceptual functions, and visual memory) compared with patients with right temporal lobe epilepsy.

Thirdly, consistent with previous reports, ${ }^{3-5}$ a significant proportion (34\%) of the patients with epilepsy studied here were depressed. Surprisingly, none of these patients with chronic and intractable epilepsy and comorbid depression had been treated with antidepressant medications on presentation to the monitoring unit. On discharge, $85 \%$ of the depressed patients were started on antidepressant drugs. Undertreatment of depression is recognised to be a common problem in the general population as well as in primary medical care populations, but less is known about the extent of undertreatment in neurological populations, including epilepsy. However, very recent evidence has suggested that underrecognition and 
undertreatment of comorbid interictal depression in chronic epilepsy is not uncommon, ${ }^{5} 378$ and the current findings reinforce that point. More attention to the degree to which comorbid psychiatric disorder is underrecognised and undertreated among patients with chronic epilepsy is needed, along with empirical study of the efficacy of subsequent treatment and impact on cognitive status.

Finally, longitudinal investigation is required to conclusively demonstrate that depression is causally associated with additional neuropsychological morbidity among patients with chronic temporal lobe epilepsy. It might be speculated that, at least in the population studied here, one possible factor linking depressed mood with impaired cognition was seizure frequency. Neuropsychological performance is affected by seizure frequency, ${ }^{39} 40$ but in the present study the adverse effects of depression on cognition remained when seizure frequency was controlled. Among the patients with left temporal lobe epilepsy there was no difference in seizure frequency with the differences in cognition between depressed and non-depressed patients evident. Alternatively, it could be speculated that more impaired cognition predisposed patients to depressed mood. These potential confounding issues can be clarified most directly in a prospective investigation, and the current results suggest that such an inquiry is indicated.

The neuropathological status of the mesial temporal lobe was comparable across depressed and non-depressed patients with left and right foci and it would seem that lateralised hippocampal pathology is not the neuropathological substrate of combined depressed mood and cognitive impairment in temporal lobe epilepsy. Other regions of the limbic system (for example, the amygdala) are possible candidates. ${ }^{61}$ The brain dysfunction underlying the identified cognitive impairment associated with depression may be at a functional (blood flow or metabolism) rather than a structural anatomical level.

In conclusion, among patients with chronic temporal lobe epilepsy, adequacy of neuropsychological functioning seems to be adversely affected by comorbid interictal depression. Although there is no greater incidence of depression in left compared with right temporal lobe epilepsy, the hypothesis is raised that neuropsychological performance may be more adversely affected in patients with left temporal lobe epilepsy. Depression in epilepsy seems to be underrecognised, or at least undertreated, and greater attention should be directed to early recognition and treatment of depression given its adverse effects on quality of life. This work was presented in part at the 10th annual meeting of
the American Neuropsychiatric Association, New Orleans, 31 January to 2 February, 1999. It is supported in part by January to 2 February, 1999. It
NARSAD and NIH grant NS37738.

1 Jones-Gotman M, Smith ML, Zatorre RJ. Neuropsychological testing for localizing and lateralizing the epileptogenic
region. In: Engel J Jr, ed. Surgical treatment of epilepsies. 2nd region. In: Engel J Jr, ed. Surgical treatment of
ed. New York: Raven Press, 1993:245-61.
2 Dodrill CB, Hermann BP, Rausch R, et al. Neuropsychological testing for assessing prognosis following surgery for epilepsy. In: Engel J Jr, ed. Surgical treatment of epilepsies. 2nd ed. New York: Raven Press, 1993:263-271.

3 Victoroff J. DSM-III psychiatric diagnoses in candidates for epilepsy surgery: lifetime prevalence. Neuropsychiatry Neuropsychol Behav Neurol 1994;7:87-97.

4 Blumer D, Montouris G, Hermann BP. Psychiatric morbidity in seizure patients on a neurodiagnostic monitoring unit. $\mathcal{F}$ Neuropsychiatry Clin Neurosci 1995;7:445-56.

5 Hermann BP, Seidenberg M, Bell B. Psychiatric comorbidity in chronic epilepsy: identification, consequences and treatment of major depression. Epilepsia 2000;41(suppl 2): $31-41$

6 Mendez MF, Cummings JL, Benson DF. Depression in epilepsy. Significance and phenomenology. Arch Neurol 1986; 43:766-70.

7 Jensen I, Larsen JK. Mental aspects of temporal lobe epilepsy. Follow-up of 74 patients after resection of a temporal lobe. F Neurol Neurosurg Psychiatry 1979;42:256-

8 Brand AN, Jolles J, Gispen- deWied C. Recall and recognition memory deficits in depression. 7 Affect Disord 1992;25: $77-86$

9 Caine ED, Yerevanian BI, Bamford KA. Cognitive function and the dexamethasone suppression test in depression. Am Psychiatry 1984;141:116-8.

10 Hart RP, Kwentus JA, Hamer RM, et al. Selective reminding procedure in depression and dementia. Psychol Aging 1987; 2:111-5.

11 King DA, Cox C, Lyness JM, et al. Neuropsychological effects of depression and age in an elderly sample: a confirmatory study. Neuropsychology 1995;9:399-408.

12 Speedie LJ, Rabins PV, Pearlson GD. Confrontation naming deficit in dementia of depression. F Neuropsychiatry Clin Neurosci 1990;2:59-63.

13 Paradiso S, Lamberty GJ, Garvey MJ, et al. Cognitive impairment in the euthymic phase of chronic unipolar depression. F Nerv Ment Dis 1997;185:748-54.

14 Bolla-Wilson K, Robinson RG, Starkstein SE, et al. Lateralization of dementia of depression in stroke patients. $A m$ F Psychiatry 1989;146:627-34.

15 Robinson RG, Bolla-Wilson K, Kaplan E, et al. Depression influences intellectual impairment in stroke patients. $\mathrm{Br} \mathcal{F}$ Psychiatry 1986;148:541-7.

16 Kessler D, Lloyd K, Lewis G, et al. Cross sectional study of symptom attribution and recognition of depression and anxiety in primary care $B M \mathcal{F} 1999 ; 318$ :436-40;

17 Simon GE, Goldberg D, Tiemens BG, et al. Outcomes of recognized and unrecognized depression in an international primary care study. Gen Hosp Psychiatry 1999;21: $97-105$

18 Hirschfeld RM. American health care systems and depression: the past, present, and the future. I Clin Psychiatry1998; 59:5-10.

19 Barge-Schaapveld DQ, Nicolson NA, Berkhof J, et al. Quality of life in depression: daily life determinants and variability. Psychiatry Res 1999;88:173-89.

20 Blume WT, Grabow JD, Darley FL, et al. Intracarotid amobarbital test of language and memory before temporal lobectomy for seizure control. Neurology 1973;23:812-9.

21 Wyler AR, Ojemann GA, Lettich E, et al. Subdural strip electrodes for localizing epileptogenic foci. 7 Neurosurg 984:60:1195-200.

22 Bear DM, Fedio P. Quantitative analysis of interictal behavior in temporal lobe epilepsy. Arch Neurol 1977;34: 454-67.

23 Beck AT, Ward CH, Mendelson M, et al. An inventory for measuring depression. Arch Gen Psychiatry 1961;76:33945.

24 Radloff LS. The CES-D scale: a self report depression scale for research in the general population. Applied Psychological Measurement 1977;1:385-401.

25 Hathaway SR, McKinley JC. The Minnesota multiphasic personality inventory (MMPI) manual. New York: Psychological Corporation, 1967.

26 Wechsler D. Wechsler adult intelligence scale: revised. New York: Psychological Corporation, 1981.

27 Jastak S, Wilkinson G. The wide range achievement test, revised: administration manual. Wilmington, DE: Jastak Associates, 1984.

28 Spreen O, Benton AL. Neurosensory center comprehensive examination for aphasia (NCCEA). Experimental edition. Iowa City, Iowa: University of Iowa, Department of Neurology, 1965.

29 De Renzi E, Vignolo L. The Token test: a sensitive test to detect receptive disturbances in aphasiscs. Brain 1962;85: $665-78$

30 Benton A L, Sivan ABdeS, Hamsher K, et al. Contributions to neuropsychological assessment. 2nd ed. New York: Oxford University Press, 1994

31 Wechsler D. Wechsler memory scale: revised. New York: Psychological Corporation, 1987.

32 Warrington EK. Recognition memory test: manual. Windsor: NFER-Nelson, 1984

33 Reitan R, Wolfson D. The Halstead-Reitan neuropsychoogical test battery: theory and interpretation. Tucson: Neuropsychology Press, 1985.

34 Heaton RK. Wisconsin card sort test. Odessa, FL: Psychological Assessment Resources, 1981.

35 Benton A L, Hamsher K. Multilingual aphasia examination. New York: Oxford University Press, 1983. 
36 Hermann BP, Wyler AR, Somes G, et al. Pathological status of the mesial temporal lobe predicts memory outcome from left anteric 652 .

37 Ettinger A, Weisbrot DM, Nolan EE, et al. Symptoms of depression and anxiety in pediatric epilepsy patients. Epilepsia 1998;39:595-9.

38 Wiegartz P, Seidenberg M, Woodard A, et al. Co-morbid psychiatric disorder in chronic epilepsy: recognition and etiology of depression. Neurology 1999;53:3-25.
39 Dikmen S, Matthews CG. Effect of major motor seizure frequency upon cognitive-intellectual functions in adults. Epi-

lepsia 1977;18:21-9.
40 Dodrill CB. Correlates of generalized tonic-clonic seizures with intellectual, neuropsychological, emotional, and social function in patients with epilepsy. Epilepsia 1986;27:399-411.

41 Csernansky JG, Leiderman DB, Mandabach M, et al. Psychopathology and limbic epilepsy: relationship to seizure variables and neuropsychological function. Epilepsia 1990;31:275-80. 